ARTICLE

\title{
Broadband decoupling of intensity and polarization with vectorial Fourier metasurfaces
}

Qinghua Song (10 1, Arthur Baroni (10) 2, Pin Chieh Wu (D) ${ }^{3}$, Sébastien Chenot ${ }^{1}$, Virginie Brandli', Stéphane Vézian (10 ${ }^{1}$, Benjamin Damilano ${ }^{1}$, Philippe de Mierry ${ }^{1}$, Samira Khadir ${ }^{1}$, Patrick Ferrand (iD ${ }^{2} \&$ Patrice Genevet ${ }^{1 凶}$

Intensity and polarization are two fundamental components of light. Independent control of them is of tremendous interest in many applications. In this paper, we propose a general vectorial encryption method, which enables arbitrary far-field light distribution with the local polarization, including orientations and ellipticities, decoupling intensity from polarization across a broad bandwidth using geometric phase metasurfaces. By revamping the well-known iterative Fourier transform algorithm, we propose "à la carte" design of far-field intensity and polarization distribution with vectorial Fourier metasurfaces. A series of non-conventional vectorial field distribution, mimicking cylindrical vector beams in the sense that they share the same intensity profile but with different polarization distribution and a speckled phase distribution, is demonstrated. Vectorial Fourier optical metasurfaces may enable important applications in the area of complex light beam generation, secure optical data storage, steganography and optical communications.

\footnotetext{
${ }^{1}$ Université Côte d'Azur, CNRS, CRHEA, Rue Bernard Gregory, Sophia Antipolis, Valbonne, France. ${ }^{2}$ Aix Marseille Univ, CNRS, Centrale Marseille, Institut Fresnel, Marseille, France. ${ }^{3}$ Department of Photonics, National Cheng Kung University, Tainan, Taiwan. ${ }^{凶}$ email: Patrice.Genevet@crhea.cnrs.fr
} 
O ptical waveform control plays a critical role in the optical systems for various applications. Among the different methods to address the electromagnetic field distribution in the far field, optical metasurfaces ${ }^{1-12}$-artificial materials that consist of subwavelength structure arrays-are capable of tailoring the waveform of the electromagnetic waves with an unpreceded level of precision. In particular, due to the versatility of this approach, it is possible to engineer both amplitude and polarization information at will. Vectorial meta-holograms with arbitrary polarization have been developed using a diatomic reflective metasurface $^{13,14}$ and a geometric phase-based metasurface ${ }^{15}$. However, the generated polarization is limited by the multiplexing metasurface's sub-pixels, which are not able to realize arbitrary spatially distributed polarization as yet. Some efforts have been made by combining geometric phase and propagation phase ${ }^{16,17}$, but it severely suffers from narrow bandwidth. A broadband wavefront control that can decouple amplitude from polarization information has yet to be demonstrated.

One of the most important applications of meta-holograms is information security, which is important in many areas of the society, such as protecting individuals, industries, and military information from leaks and stealing. Among the different communication channels and information sharing techniques, photonics is the most efficient and effective way of carrying information across long distances. Optical waveforms possess many degrees of freedom, such as amplitude, phase, frequency, and polarization, and each can be used for data encoding. Moreover, optical encoding methods require specific professional equipment for data encoding, providing a more secure way towards high-security information encoding. Various optical encoding methods have been developed based on the intensity, such as spatial correlators ${ }^{18}$, optical exclusive or (XOR) image encryption ${ }^{19}$, phase-shifting interferometry ${ }^{20}$, polarization-dependent images ${ }^{21,22}$, Lippmann plate $^{23}$, and holograms ${ }^{24,25}$. Many other efforts of optical encoding have been made by using multiplexing meta-hologram that can encode the optical information into multi-channels of holographic images ${ }^{26-30}$. The most common approach is based on polarization-dependent meta-hologram, which creates different holographic images using different polarizations of the incident beam ${ }^{31-36}$. Chiral metaholograms are also introduced for direction-dependent holographic encoding ${ }^{37-40}$. Other encoding methods relying on incident wavelength $^{41}$, nonlinear effect ${ }^{42}$, spatial frequency ${ }^{43}$, orbital angular momentum $^{44-46}$, and tunable metasurface ${ }^{47-49}$ are also demonstrated. It is noteworthy that all of these proposed multiplexing meta-holograms encode information on the intensity of the holographic images.

In this study, we propose a vectorial Fourier metasurface for which amplitude and polarization information can be addressed independently one from the other. We utilize this specificity to encode intensity and two polarization information channels, namely ellipticity and azimuth, to produce far-field decoupled images. The image refers to the spatial distribution of either total intensity, ellipticity, or azimuth information. The design of the metasurface is realized using a modified iterative Fourier transform (IFT) algorithm that does not only consider far-field amplitude but also the far-field polarization spatial distribution. Our calculation results consist of discretized transmission matrices representing the spatial amplitude and polarization into pixelated profiles. The design of the nanostructured interfaces capable of matching these distributions therefore requires precise control of all properties of transmitted light. To this end, we composed arbitrary amplitude, phase, and polarization pixels by superposing two amplitude-modulated and phase-delayed beams with the opposite circular polarizations (CPs).

With respect to previously proposed methods, our approach defines the metasurface capability by considering the polarization distribution in the far field only, i.e., as a result of the propagation leading to left CP (LCP) and right CP (RCP) far fields. As the metasurface plane is encoded via the Fourier transformation of targeted fields, the realization of optical information encoding in this work is completely outperforming than that of previous demonstrations with multiplexing ${ }^{15}$. As a proof of principle, we designed a series of far-field intensity profiles presenting a given donut-like intensity distribution structured with different polarization orientations. Interestingly, these vectorial fields look like the well-known cylindrical vector beams (CVBs; previously discussed in the literature). Nevertheless, they differ strongly to CVB in the sense that, even if the polarization is maintained, their long-range far-field phase distribution is lost during the optimization process. Roughly speaking, the randomization of the farfield phase using IFT techniques produces granular intensity distributions or intensity-modulated speckle signals, which match the overall targeted CVB donut intensity and polarization. These field profiles are extremely interesting, as they are mixing longrange transverse coherence (correlation between the fields at different points) with very short-range spatial phase correlation (the degree to which the spatial phase are related). In addition, we demonstrate a class of optical interfaces that encodes the orientation angle and ellipticity angle of the polarization in a uniformly distributed intensity profile. To resolve the encoded information, we use both conventional Stokes parameter measurements and vectorial ptychography to characterize both metasurface and their far-field complex amplitudes.

\section{Results}

Design method. The design principle of the vectorial Fourier metasurface is shown in Fig. 1. Each pixel of the metasurface consists of four lines of phase gradient supercells as shown in Fig. 1a, in which the top two lines and bottom two lines of metastructures are arranged counter-clockwise and clockwise, respectively, with the same orientation increment angle of $\delta_{\mathrm{d}}$. Each building block of the pixels, the pillar meta-structure, acts as a half-waveplate that converts the handiness of the incident $\mathrm{CP}$ beams and imposes a geometry phase (also called Pancharatnam-Berry (PB) phase) of $\pm 2 \delta$, where $\delta$ is the orientation angle of each pillar (the signs "-" and " + " denoted clockwise and counter-clockwise rotation, respectively), i.e., $|+\rangle \rightarrow e^{i 2 \delta}|-\rangle$ and $|-\rangle \rightarrow e^{-i 2 \delta}|+\rangle$, where $|+\rangle$ represents LCP and $|-\rangle$ represents RCP. Considering that the incident linear polarized (LP) light can be decomposed into LCP and RCP, the clockwise lines in a pixel deflect the LCP light to RCP light with a deflection angle of $\theta_{\mathrm{t}}=\arcsin \left(\frac{2 \delta_{\mathrm{d}}}{k_{0} P}\right)$ as shown in Fig. $1 \mathrm{~b}$, where $k_{0}$ is the wavenumber in the free space and $P$ is the period of the unit cell. The counter-clockwise lines in the same pixel deflect the RCP to LCP at the same angle of $\theta_{\mathrm{t}}$. The starting orientation angle of the four lines from top to bottom are $\delta_{+}, \delta_{+}+\Delta \delta_{+}, \delta_{-}$, and $\delta_{-}+\Delta \delta_{-}$, where $\Delta \delta_{+}$and $\delta_{+}$are respectively used to control the relative amplitude and phase between LCP and RCP. We ignore the co-polarization in the following text, simply because it is diffracted at the zero order and it does not interfere with the cross-polarized fields. The complex amplitude $a^{m}$ in the metasurface plane is given by,

$$
a^{m}\left(x^{m}, y^{m}\right)=A_{+}^{m}\left(x^{m}, y^{m}\right) e^{i \varphi_{+}^{m}\left(x^{m}, y^{m}\right)}+A_{-}^{m}\left(x^{m}, y^{m}\right) e^{i \varphi_{-}^{m}\left(x^{m}, y^{m}\right)}
$$

where the superscript $m$ represents the metasurface plane, $x^{m}$ and $y^{m}$ represent the pixel positions in the metasurface plane, and $A_{ \pm}^{m}\left(x^{m}, y^{m}\right)$ and $\varphi_{ \pm}^{m}\left(x^{m}, y^{m}\right)$ are the amplitude and phase of pixel $\left(x^{m}, y^{m}\right)$ at the metasurface plane generated by the two CP of the light beam. For simplicity, in the following we ignore the notation 

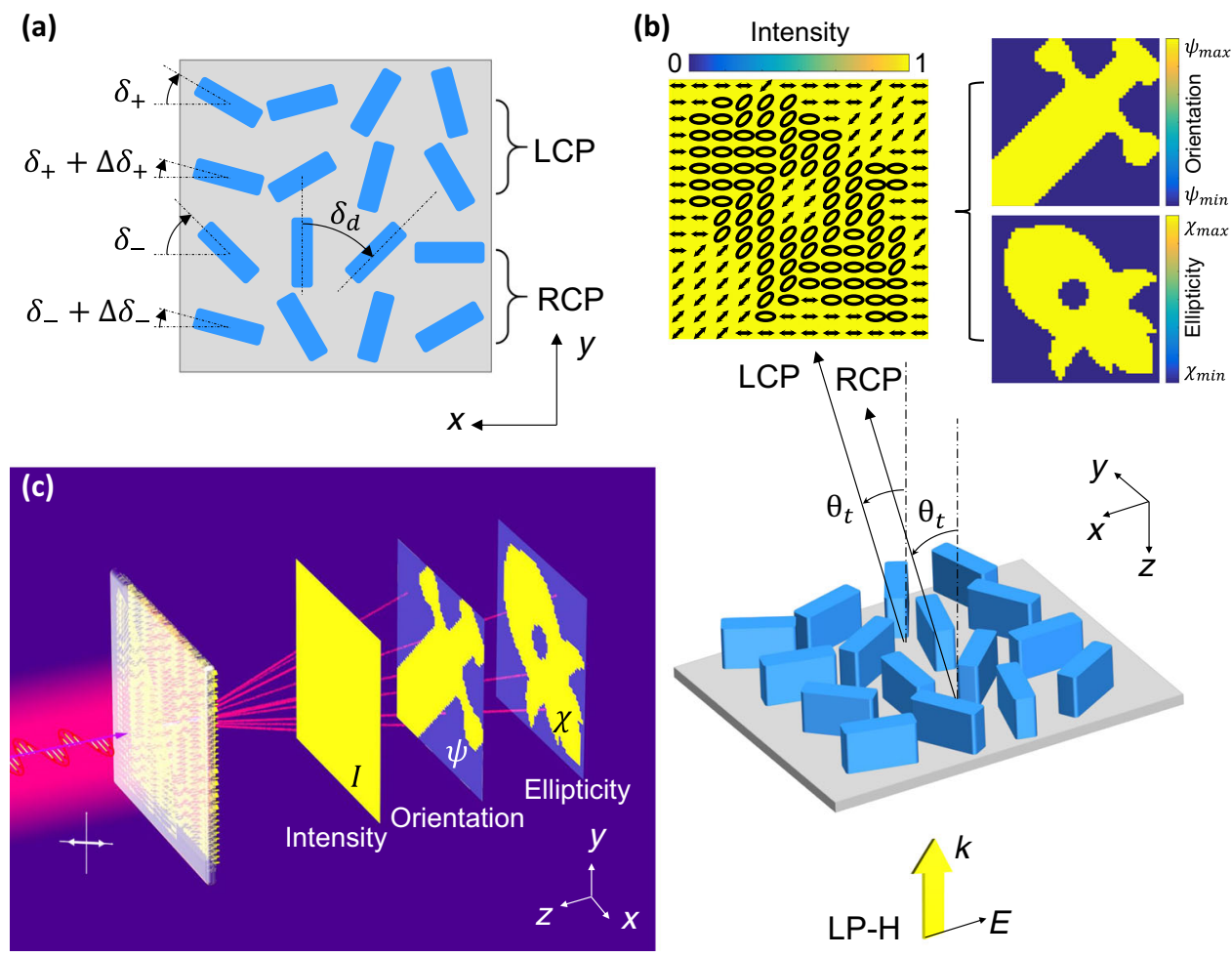

Fig. 1 Design principle of vectorial Fourier metasurface for arbitrary far-field light distribution of intensity and polarization. a Top view of one pixel of the metasurface. Arbitrary polarization requires superposition of two orthogonal polarizations (chosen here as RCP and LCP) with controllable relative amplitudes and phases. To do so, each CP state is produced by two lines of the same handiness with different SOA of $\triangle \delta_{ \pm}$to control the relative amplitude and $\delta_{ \pm}$to control the relative phase. $\mathbf{b}$ Perspective view of the metasurface and far-field light distribution. The LP input light can be decomposed into two $\mathrm{CP}$ beams, which are deflected to the same angle of $\theta_{\mathrm{t}}$. The holographic phase information is encoded in the LCP and RCP independently, so that arbitrary polarization is realized by the superposition of the two CP beams. c Schematic of the intensity and polarization decoupling using vectorial Fourier metasurface. The orientation angle and ellipticity of the polarization exhibiting a "Blade" and a "Rocket" images are encoded in a uniformly distributed intensity profile.

of $\left(x^{m}, y^{m}\right)$. The amplitude $A_{ \pm}^{m}$ is controlled by the rotation angle difference of $\triangle \delta_{ \pm}$due to the interference between two lines of LCP (or RCP) as,

$$
A_{\sigma}^{m}=\left|e^{-i 2 \sigma \delta_{\sigma}}+e^{-i 2 \sigma\left(\delta_{\sigma}+\Delta \delta_{\sigma}\right)}\right| / 2=\sqrt{\left(1+\cos 2 \Delta \delta_{\sigma}\right) / 2}
$$

where $\sigma=+($ or +1$)$ represents LCP and $\sigma=-($ or -1$)$ represents RCP. The phase $\varphi_{ \pm}^{m}$ is generated by the rotation angle of $\delta_{ \pm}$, thanks to the geometric phase as,

$$
\varphi_{\sigma}^{m}=-2 \sigma \delta_{\sigma}
$$

Therefore, by varying the value of $\delta_{ \pm}$and $\Delta \delta_{ \pm}$, arbitrary amplitude and phase information in the metasurface plane can be assigned to each pixel independently from the others, so as to control far-field amplitude and polarization information at will.

To decouple amplitude from far-field polarization information, we propose modifying the conventional Gerchberg-Saxton (GS) algorithm to a version working for vectorial fields. The GS utilizes IFT as shown in Fig. 2 (see more details in Supplementary Note 1$)^{17,26,27}$, and in its vectorial version-instead of converging to a phase profile in the metasurface plane-we consider the phase profiles of two CP beams noted $\varphi_{\sigma}^{m}$ realized by rotating the angle of $\delta_{\sigma}$ according to Eq. 3. In this implementation, the farfield polarization can be controlled over the entire profile, despite the fact that GS converges to designs with randomly distributed far-field phase profile. The condition for far-field polarization addressing requires that the phase retardation between orthogonal polarization channels is properly adjusted, i.e., the phase value for both polarization channels is randomly distributed on the transverse plane with a controllable phase retardation.

For a convenience purpose, we keep the amplitude in the metasurface plane $A_{+}^{m}$ uniform for all pixels, i.e., $\Delta \delta_{+}$and $\Delta \delta_{-}$ are two constant values for all pixels determined by the total intensity of two CP beams $I_{ \pm}^{m}$. The latter are calculated considering the intensity integral of all pixels in the image plane as: $I_{ \pm}^{m}=I_{ \pm}^{f}=\sum_{x, y=1}^{N_{x}, N_{y}}\left(a_{ \pm}^{f}\left(x^{f}, y^{f}\right)\right)^{2}$, where the superscript $f$ represents the far-field image plane, $\left(x^{f}, y^{f}\right)$ represent the pixel position in the far-field image plane, $N_{x} \times N_{y}$ is the total pixel number, and $a_{ \pm}^{f}\left(x^{f}, y^{f}\right)$ are the amplitude of LCP and RCP light of each pixel $\left(x^{f}, y^{f}\right)$. It can be shown that the rotation angles $\Delta \delta_{ \pm}$are given by (see more details in Supplementary Note 2),

$$
\begin{cases}\Delta \delta_{\sigma}=0, & \text { if } I_{\sigma}^{f} \geq I_{-\sigma}^{f} \\ \Delta \delta_{\sigma}=\operatorname{acos}\left(\frac{2 \sum_{x, y=1}^{N_{x}, N_{y}}\left(a_{\sigma}^{f}\left(x^{f}, y^{f}\right)\right)^{2}}{\sum_{x, y=1}^{N_{x}, N_{y}}\left(a_{-\sigma}^{f}\left(x^{f}, y^{f}\right)\right)^{2}}-1\right) / 2, & \text { if } I_{\sigma}^{f}<I_{-\sigma}^{f}\end{cases}
$$

Equations (3) and (4) are then used to recover the orientation angles of each pixel of the metasurface.

The meta-structures are simulated using full-wave finitedifference time-domain (FDTD) and the simulation results are shown in Fig. 3. The top view and perspective view of one metastructure are shown in Fig. 3a, b, respectively. One-micrometertall GaN nanopillars, grown on low-index lattice-matched Sapphire substrate, are realized with rectangular cross-sections to induce structural birefringence. Both $\mathrm{GaN}$ and sapphire are 


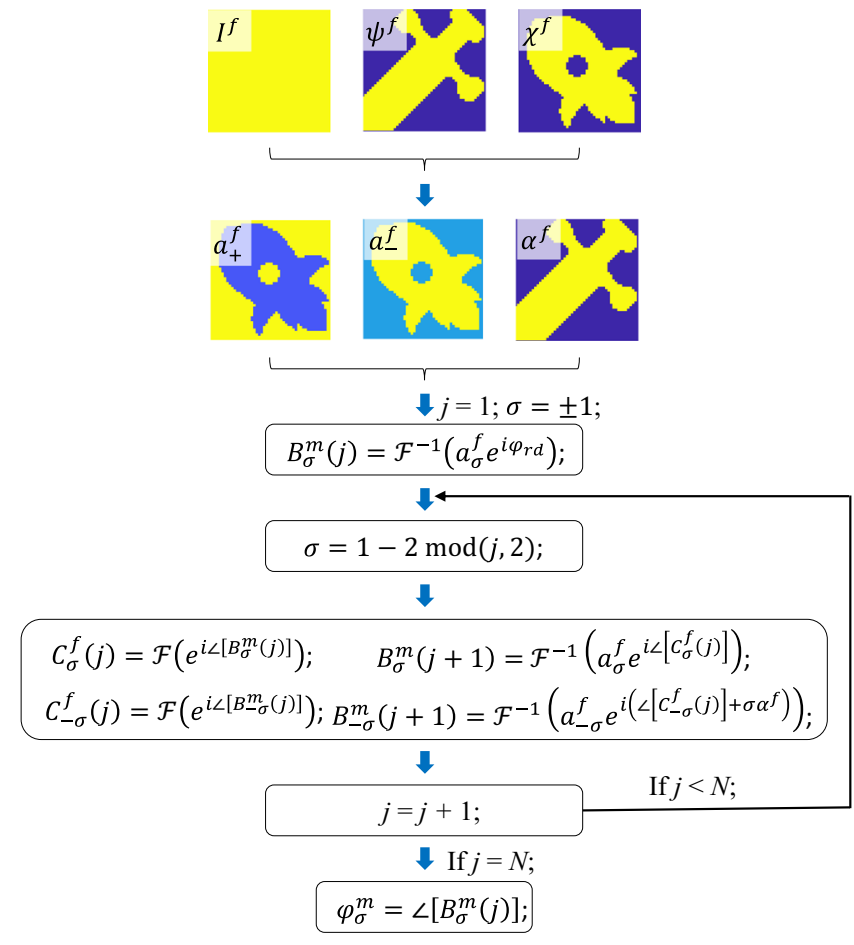

Fig. 2 Modified iterative Fourier transform algorithm. With respect to intensity-only iterative Fourier transform algorithm, the current version considers several input information to realize diffraction patterns with arbitrary intensity, azimuth, and ellipticity angles of the polarization. The algorithm converges to a vectorial profile optimizing the amplitude of both $\operatorname{LCP}\left(a_{+}^{f}\right)$ and RCP $\left(a_{-}^{f}\right)$, and the phase difference between the two CP beams $\left(\alpha^{f}\right)$. The notation $\sigma$ represents the handiness of the CP beam, where + or +1 represents LCP and - or -1 represents RCP. A random phase of $\varphi_{r d}$ is used for the starting phase. The number of iterations is $N=$ 100. The final holographic phase of the metasurface is $\varphi_{\sigma}^{m}$. The superscript $m$ indicates the metasurface plane and $f$ is the image plane in the far field (see more details in Supplementary Note 1).

transparent in the entire visible range, which are perfect candidates for the design of visible optical metasurfaces. The period of the nanostructure unit cell is $P=300 \mathrm{~nm}$ to avoid spurious diffraction effects in the substrate. The width is fixed to $L_{\mathrm{v}}=120 \mathrm{~nm}$. The CP conversion efficiency is shown in Fig. 3c with the long axis of the nanopillar $L_{\mathrm{u}}$ swept from 160 to $260 \mathrm{~nm}$ and the wavelength $\lambda$ swept from 450 to $700 \mathrm{~nm}$. The dash line indicates the CP conversion at $L_{\mathrm{u}}=210 \mathrm{~nm}$, where the CP conversion efficiency is higher than $50 \%$ across almost the entire visible range. Figure 3d, e show the electric field distribution along short and long axis of the pillar, respectively, at the point of $L_{\mathrm{v}}=120 \mathrm{~nm}$ and $\lambda=575 \mathrm{~nm}$ (the purple star in Fig. 3c). It is shown that there are 5.5 and 5 oscillations of electric field in $E_{x}$ and $E_{y}$ in the $\mathrm{GaN}$ nanopillar, i.e., a signature of half a wavelength retardation difference, which verifies that the meta-structure acts as a nanoscale half-waveplate for these structural parameters and operation wavelength. In addition, when the nanopillar is rotated with an angle of $\delta$, a geometric phase of $2 \delta$ is obtained on cross$\mathrm{CP}$ as shown in Fig. 3f. The simulated geometric phase by using FDTD shown in blue stars agrees well with the theoretical one $\left(\varphi_{\mathrm{RL}}=2 \delta\right)$ shown by the red curve. In addition, the $\mathrm{CP}$ conversion efficiency between LCP and RCP is, as expected, near unity as shown by the black curve.
In order to validate our approach to decouple intensity from polarization, we conceived a series of spatially variant far-field polarization profiles distributed on a donut far-field intensity profile (with the same radius), but having different azimuthal angle of the linear polarization defined by $\psi(x, y)=$ $l \varphi(x, y)+m \pi / 2$, where $l$ is an integer number that represents the turns of the polarization rotation encircling the donut intensity profile, $x$ and $y$ are the coordinate of beam, $\varphi(x, y)=\tan ^{-1}\left(\frac{y}{x}\right), m=0$ represents the radial mode, and $m=$ 1 represents the azimuthal mode. We demonstrated four designs with different combinations of $m$ and $l$ (see more details in Supplementary Fig. 3). The scanning electron microscope images of the fabricated vectorial beam metasurfaces are shown in the first row of Fig. 4. The second row represents the designed intensity and polarization profiles. The measured total intensity profiles are shown in the third row, which agree well to the designed intensities. By placing a linear polarizer with different rotation angles in front of the vectorial beams, different patterns are observed from the fourth to the seventh rows. Interestingly, even if these beams resemble the well-known CVBs, we prove that they do not feature long-range spatial phase correlation. To do so, the amplitude and polarization information of the vectorial farfield patterns were also investigated by means of vectorial ptychography. This computational microscopy technique can indeed provide a quantitative map of the metasurface, thanks to the retrieval of its Jones matrix ${ }^{50}$, mapped at a microscopic resolution of the whole sample area (see "Methods" and Supplementary Fig. 2b). This quantitative knowledge of the metasurface optical properties makes it possible to model totally the far-field patter, by modeling a horizontally polarized illumination on the sample, followed by propagation (see Supplementary Fig. 2c). The resulting far-field complex amplitude distributions, either on a LP or on an RCP/LCP decomposition basis, as shown in Fig. 5, exhibit a short spatial phase correlation with a clear speckle patter, while maintaining polarization over the whole intensity pattern. Indeed, with respect to $\mathrm{CV}$ beams that are vectorial solutions of Maxwell's equations obeying axial symmetry in both amplitude and phase, our solution to produce spatially distributed amplitude and polarization field does not impose long-range spatial phase correlation. These fields could be beneficial for practical applications in laser machining, remote sensing, and so forth ${ }^{51,52}$, or to decouple phase and polarization in singular optics. Using PB phase-tuning mechanisms, the polarization encoding is simply given by the rotation angle of the nanostructures, resulting in a broad operating bandwidth. It reveals that broad operating bandwidth is generally not achievable with a combination of propagation and $\mathrm{PB}$ phases ${ }^{16}$. The characterization of the broadband properties is shown in Fig. 6. A $\mathrm{CV}$ beam with $l=-2$ and $m=0$ is measured from $\lambda=475 \mathrm{~nm}$ to $\lambda=675 \mathrm{~nm}$. A donut intensity profile is shown in the first row without polarizer. Subsequently, we insert a linear polarizer in front of the image. The same pattern is observed for all of the wavelength with fixed transmission axis of polarizer as shown from the second to fifth rows, indicating that the metasurface could maintain polarization distribution properties over a broad wavelength range. The efficiency of the $\mathrm{CV}$ beam is in the range of $5 \sim 17 \%$ across the entire visible range as shown in Supplementary Fig. 5, which is a bit low due to the fabrication errors and large pixel size. The latter is larger than the operating wavelength, resulting in higher-order images, which decreases the efficiency of the interested order.

After verification of the design approach with simple vectorial beam, we propose to encode optical information relying on the azimuth and ellipticity angles of the polarization information rather than encoding intensity profiles of conventional polarization 
(a)

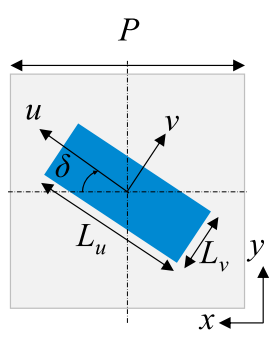

(b)

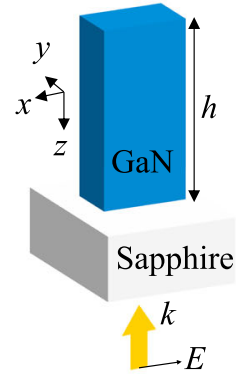

(c)

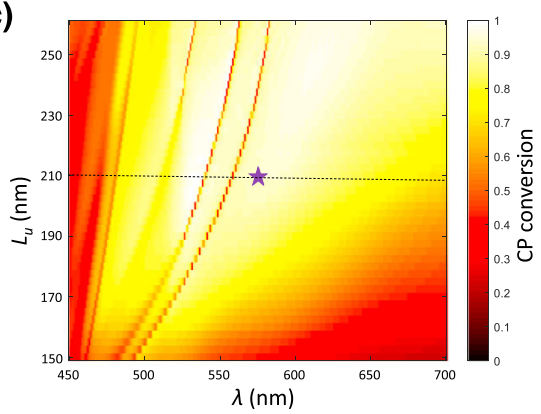

(d)

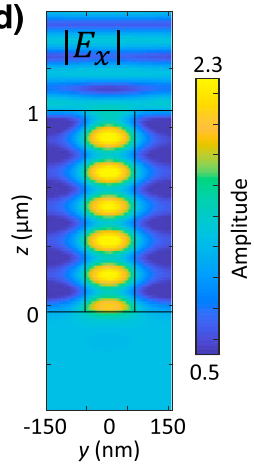

(e)

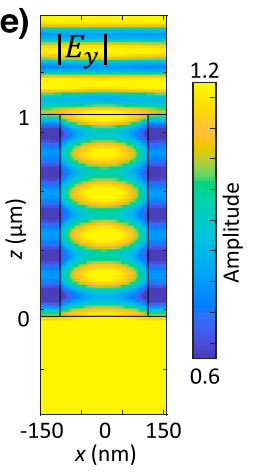

(f)

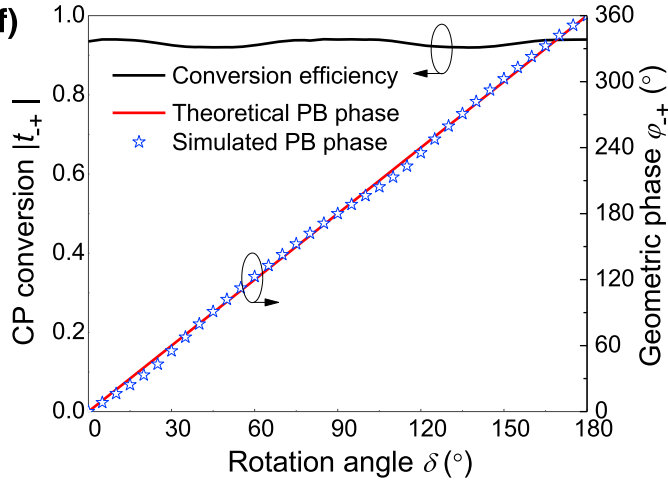

Fig. 3 Simulated results of metasurface with birefringent GaN nanopillars on sapphire substrate with dimension of $\boldsymbol{P}=\mathbf{3 0 0} \mathbf{n m}$ and $\boldsymbol{h}=\mathbf{1} \boldsymbol{\mu m}$. a Top view and $\mathbf{b}$ perspective view of one meta-structure. The numerical calculation is performed on unit cell of $\mathrm{GaN}$ nanopillar on sapphire substrate considering periodic boundary conditions on $x$ and $y$. The width of the GaN nanopillar is fixed as $L_{v}=120 \mathrm{~nm}$. c The simulated CP conversion by sweeping the length of the GaN nanopillar $L_{u}$ from 150 to $260 \mathrm{~nm}$ in the wavelength range from 450 to $700 \mathrm{~nm}$ with zero rotation angle. The dash line represents the length of $L_{u}=210 \mathrm{~nm}$. The purple star represents the wavelength at $\lambda=575 \mathrm{~nm}$, which is the chosen point in $\mathbf{d}-\mathbf{f}$. $\mathbf{d}$ The simulated electric field distribution $E_{x}$ at the plane of $x=0$ and $\mathbf{e} E_{y}$ at the plane of $y=0$. $\mathbf{f}$ By rotating the GaN nanopillars with an angle of $\delta$ from 0 to $180^{\circ}$, a near-unity $\mathrm{CP}$ conversion is shown in the black curve and a geometric phase from 0 to $360^{\circ}$ is obtained. The simulated results are represented by the blue star, in very good agreement with the expected PB phase calculation (red curve).

states, realizing a sort of holographic polarization-only encoding technique. Two meta-holograms with the same intensity profile but different azimuth and ellipticity angles of the polarization are designed as shown in the Supplementary Fig. 6. The fabricated results of the two metasurfaces are shown in Supplementary Fig. 7. As the information is only encoded on the polarization properties, i.e., spatial distribution of the orientation and ellipticity, an additional retrieval method based on local Stokes polarimetry is required $^{53,54}$. Stokes parameters, which include the optical quantities of interest, are generally obtained using two sets of measurements, cascading a waveplate with the phase difference between fast axis and slow axis of $\phi$, and a linear polarizer with rotation angle of $\theta$ with respect to the $x$-axis in front of the image. The measured intensity profiles after the cascaded waveplate and linear polarizer are related to $\phi$ and $\theta$, which are denoted as $I(\theta, \phi)$. Therefore, the measured azimuth and ellipticity angles of the polarization are described as (see more details in Supplementary Note 3),

$\psi=\frac{1}{2} \tan ^{-1}\left(\frac{2 I\left(45^{\circ}, 0^{\circ}\right)-I\left(0^{\circ}, 0^{\circ}\right)-I\left(90^{\circ}, 0^{\circ}\right)}{I\left(0^{\circ}, 0^{\circ}\right)-I\left(90^{\circ}, 0^{\circ}\right)}\right)\left(-\frac{\pi}{2}<\psi \leq \frac{\pi}{2}\right)$

$\chi=\frac{1}{2} \sin ^{-1}\left(\frac{I\left(0^{\circ}, 0^{\circ}\right)+I\left(90^{\circ}, 0^{\circ}\right)-2 I\left(45^{\circ}, 90^{\circ}\right)}{I\left(0^{\circ}, 0^{\circ}\right)+I\left(90^{\circ}, 0^{\circ}\right)}\right)\left(-\frac{\pi}{4}<\chi \leq \frac{\pi}{4}\right)$

In additional to these measurements, the total intensity profiles, i.e., with spatially varying polarization distribution, are measured directly, without any waveplate and/or polarizer. The measured results of the first metasurface are shown in Fig. $7 \mathrm{a}-\mathrm{c}$.
As expected from the design, a uniform intensity profile is observed in Fig. 7a. However, both a "Blade" and a "Rocket" images are shown when looking at the polarization spatial distribution in the azimuth and ellipticity angles, respectively. Another design with the same uniform intensity profile but a "Tree" and a "Squirrel" polarization information is obtained in Fig. 7d-f. Besides, we also used vectorial ptychography to map both the amplitude and polarization information of the vectorial far-field patterns (see more details in "Methods" and Supplementary Fig. 2b). The Jones matrix maps of the metasurface as retrieved by vectorial ptychography are shown in Supplementary Fig. 8, whereas the far field is shown in Supplementary Fig. 9, including intensity and polarization information. As expected, a uniformly distributed intensity profile is observed in both designs of Supplementary Fig. 9a, d. Moreover, in the azimuth angle and ellipticity angle of polarization map, images of "Blade" and "Rocket" are respectively obtained in Supplementary Fig. 9b, c and images of "Tree" and "Squirrel" are observed in Supplementary Fig. 9e, f.

\section{Discussion}

In conclusion, we have demonstrated a general method to design vectorial Fourier metasurfaces, which decouple intensity from polarization information, such that spatially distributed full polarization profiles with arbitrary intensity distribution can be realized. The vectorial Fourier metasurfaces are conceived using a modified IFT algorithm that optimizes the transmission information properties to encode simultaneously both intensity and polarization far-field distribution. We produce an interesting series of far-field beam profile with donut-like intensity and spatially distributed polarization, resembling CVBs, but with 
(a)
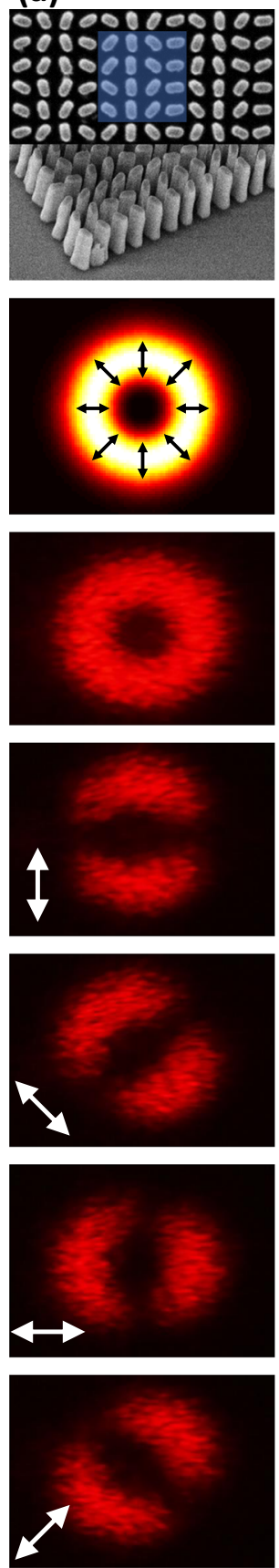

(b)
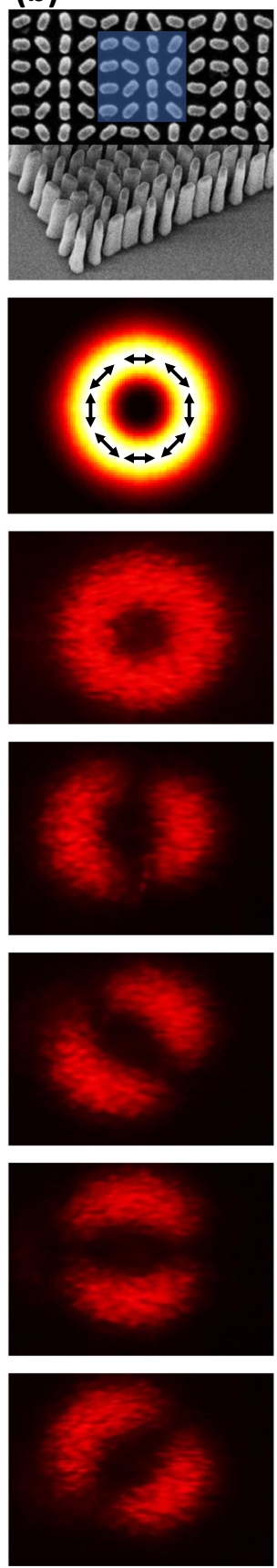

(c)
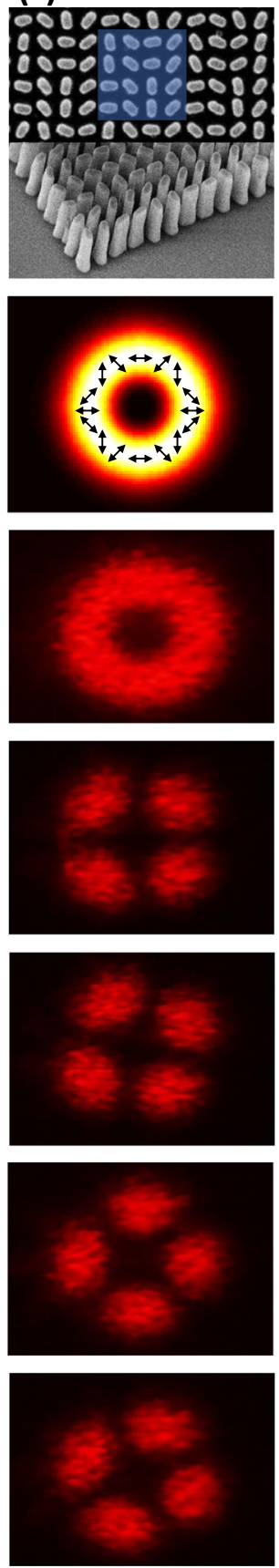

(d)
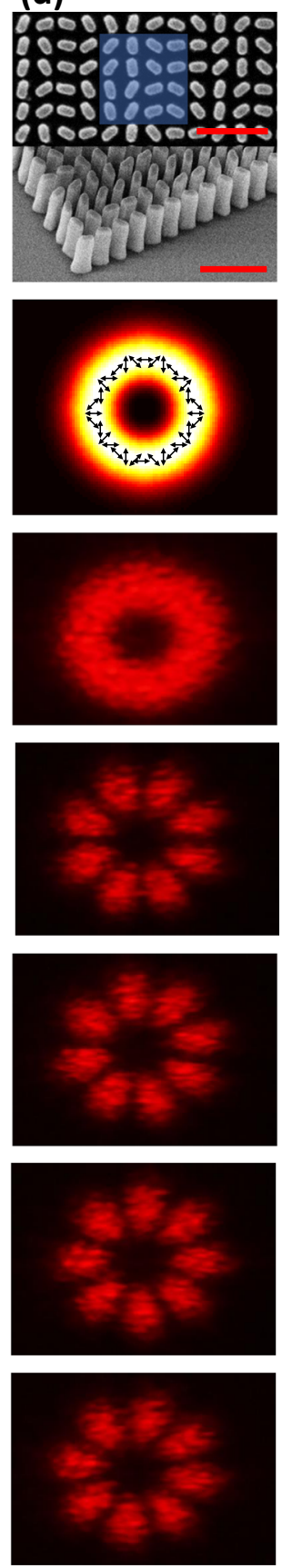

Fig. 4 Fabricated results and optical measurement of the Stokes polarization parameters of the donut polarization-distributed field profile. Field distribution with $\mathbf{a} I=1, m=0 ; \mathbf{b} I=1, m=1 ; \mathbf{c} I=-2, m=0 ; \mathbf{d} I=-4, m=0$. Top row: fabricated results with the top view (top panels) and tilt view (bottom panels). The blue area shows one pixel of the meta-hologram. The red scale bar represents $1 \mu \mathrm{m}$. Second row: intensity profiles of the designed vectorial fields. The black arrow represents the local polarization. Third row: measured intensity profiles of the field distributions without linear polarizer. Fourth to seventh rows: measured intensity profiles of the field distributions with a linear polarizer. The white arrow represents the transmission axis of the linear polarizer.

randomly distributed far-field phase distribution. To fully characterize the optical response of our Fourier metasurfaces, we retrieve the complete Jones matrix of the metasurface using vectorial ptychography and proved the short-range phase correlation in contrast to the long-range polarization distribution, indicating that both polarization channels have spatially correlated phase profiles. Furthermore, the proposed vectorial Fourier metasurfaces are able to encode complex polarization information onto uniform distributed intensity profiles. We demonstrated that a "Blade" (or "Tree") and a "Rocket" (or "Squirrel") images can be multiplexed and separately decrypted from the orientation angle and ellipticity angle of the polarization on a uniformly distributed intensity profile of a holographic image. Vectorial Fourier encoding could highly enhance the information security, having various promising applications in data encryption, optical ID tags for authentication and verification, and high-density optical data storage, but also for specific applications including optical trapping and laser machining. 

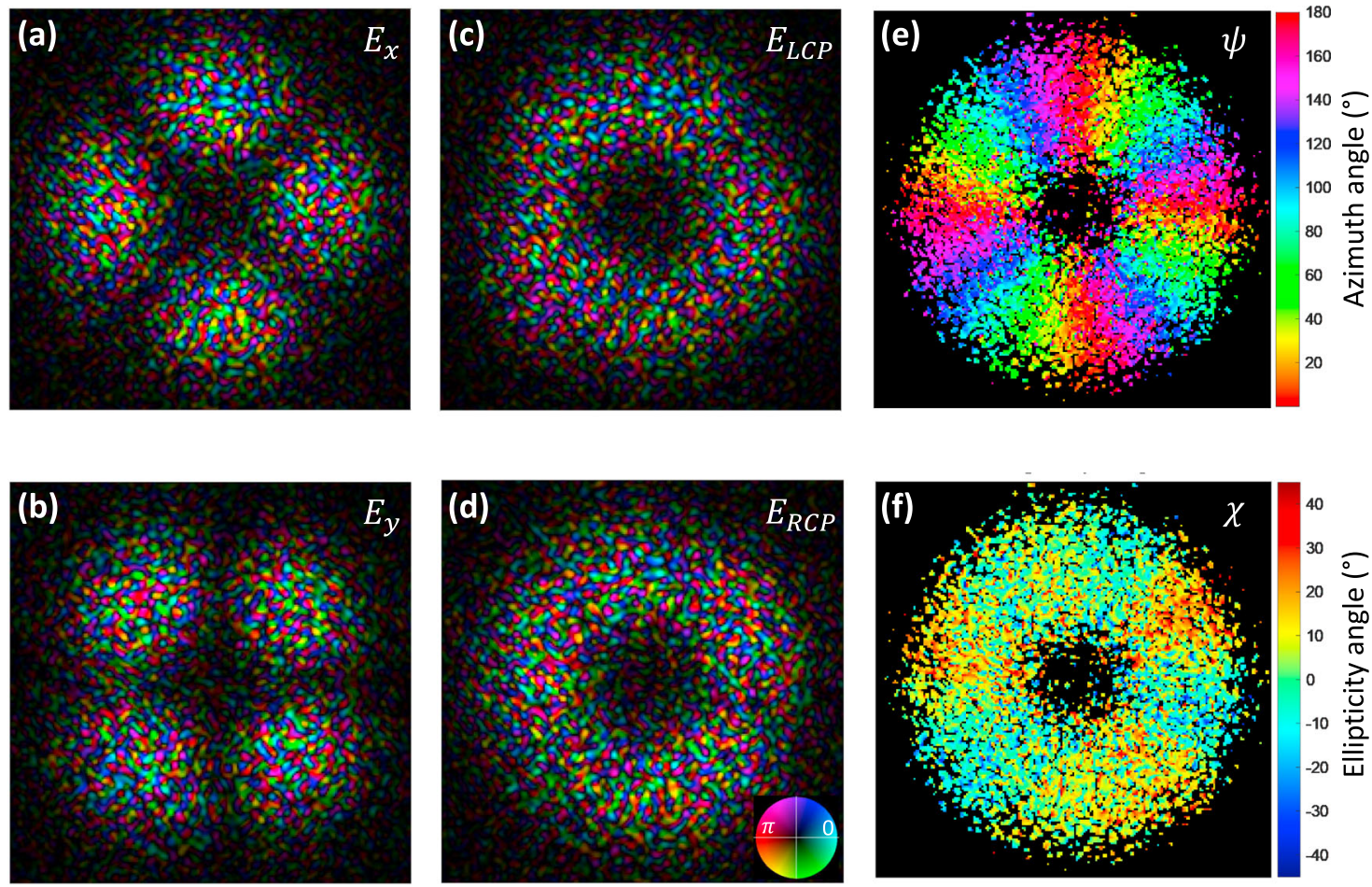

Fig. 5 Complex amplitude and polarization information of far field as retrieved by the ptychographic measurement. Measured complex amplitude of a $x$-pol., b $y$-pol., $\mathbf{c L C P}$, and $\mathbf{d}$ RCP components. The phase profiles for the four polarization components show typical speckle phase distributions. The inset figure in $\mathbf{d}$ is the color bar with phase encoded as hue and amplitude as brightness. Measured $\mathbf{e}$ azimuth angle and $\mathbf{f}$ ellipticity angle of polarization information.

(a) $\lambda=475 \mathrm{~nm}$
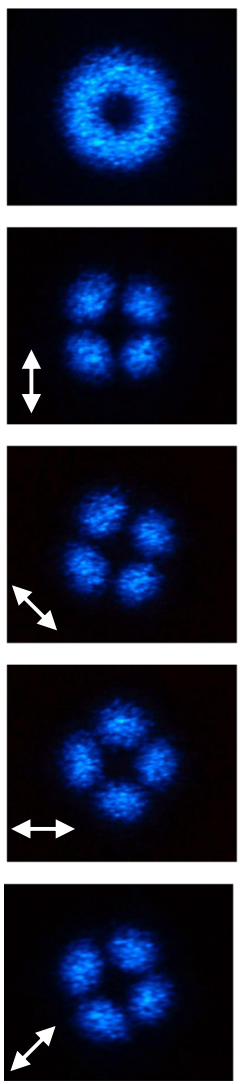

(b) $\lambda=525 \mathrm{~nm}$
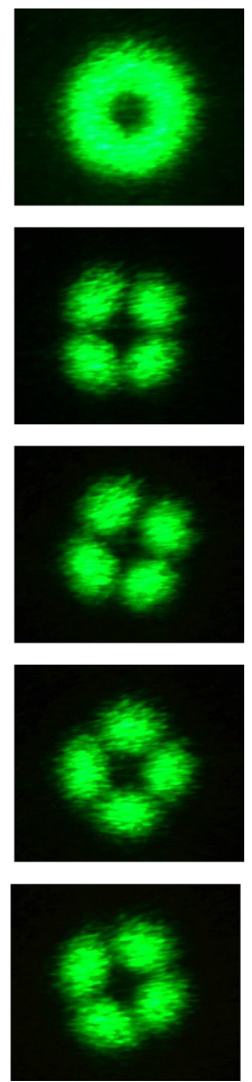

(c) $\lambda=575 \mathrm{~nm}$

(d) $\lambda=625 \mathrm{~nm}$

(e) $\lambda=675 \mathrm{~nm}$
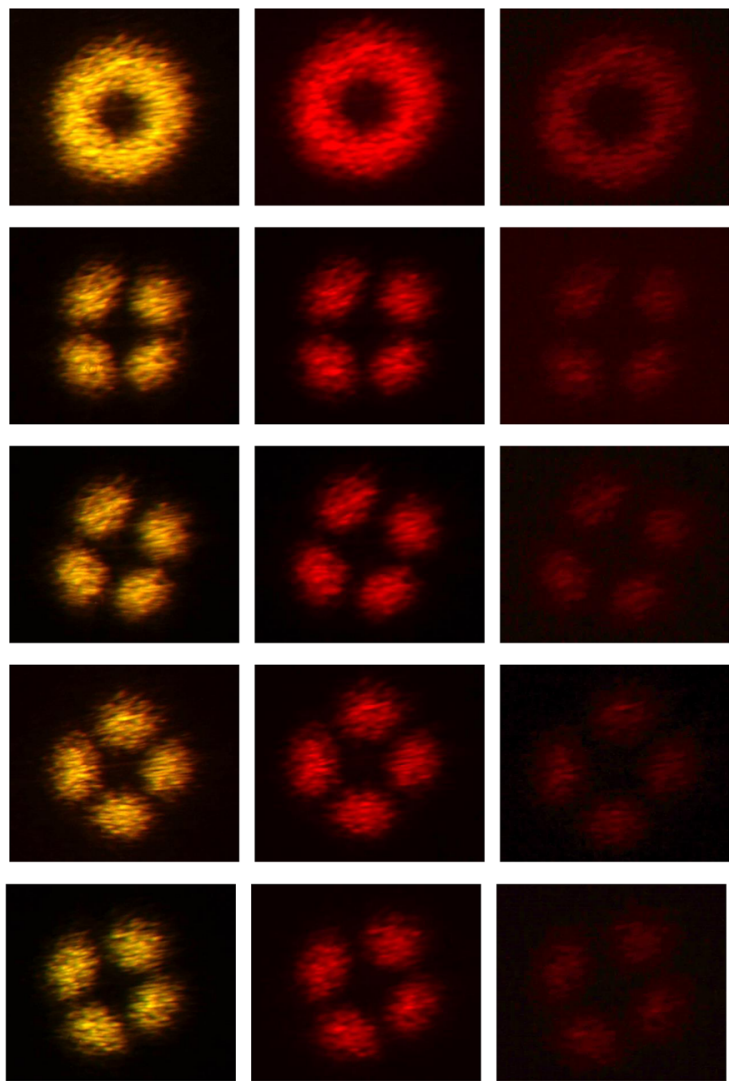

Fig. $\mathbf{6}$ Broadband characterization of the vectorial metasurfaces designed for a field profiles with $\boldsymbol{I}=-\mathbf{2}, \boldsymbol{m}=\mathbf{0}$. The measured intensity profiles at different wavelengths of $\mathbf{a} \lambda=475 \mathrm{~nm}, \mathbf{b} \lambda=525 \mathrm{~nm}, \mathbf{c} \lambda=575 \mathrm{~nm}, \mathbf{d} \lambda=625 \mathrm{~nm}$, e $\lambda=675 \mathrm{~nm}$. Top row: measured intensity profiles without linear polarizer. Second to fifth rows: measured intensity profiles with a linear polarizer. The white arrow represents the transmission axis of the linear polarizer. 
(a)

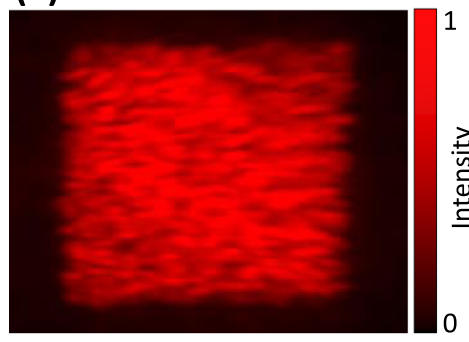

(d)

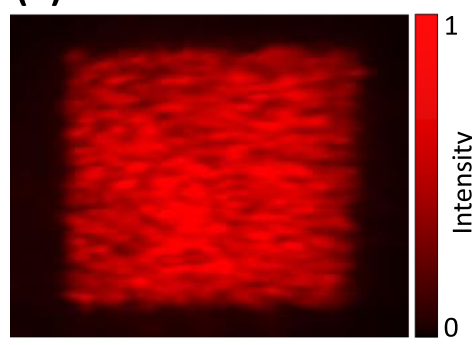

(b)

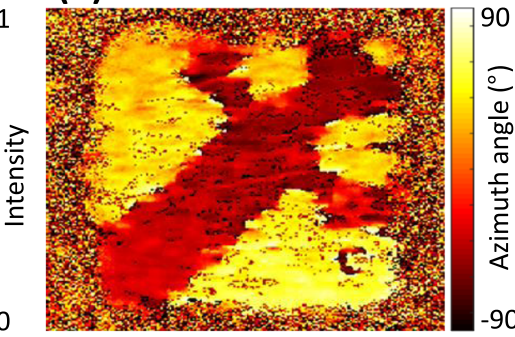

(e)

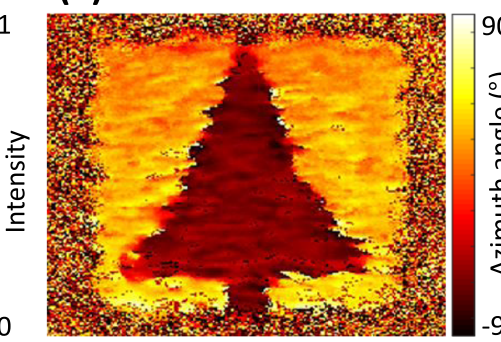

(c)

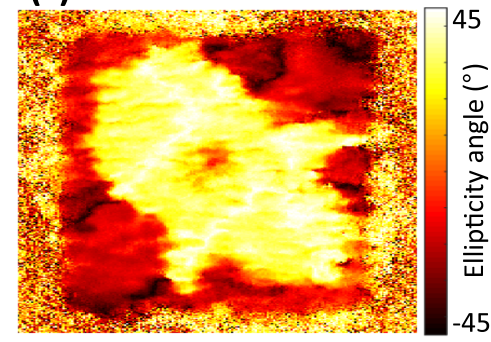

(f)

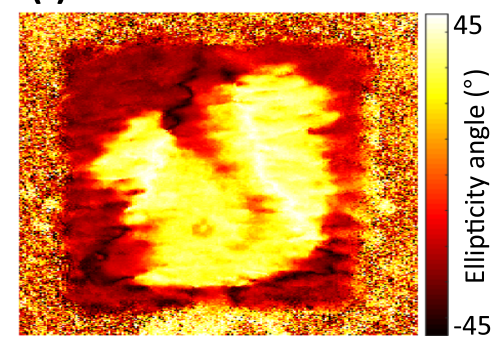

Fig. 7 Measurement results of vectorial encoded images. a-c Show the data obtained for the first metasurface design. $\mathbf{d}-\mathbf{f}$ Correspond to the data for the second metasurface design. a-f Measurement results using conventional optical setup to image the Stokes parameters. a, $\mathbf{d}$ Measured intensity; $\mathbf{b}, \mathbf{e}$ azimuth angle; $\mathbf{c}, \mathbf{f}$ ellipticity angle of the polarization. Both interfaces encode a similar uniformly distributed intensity profile as shown in $\mathbf{a}$ and $\mathbf{d}$. Colorcoded images displaying the ellipticity and the orientation; images reveal a "Blade," a "Rocket," a "Tree," and a "Squirrel" encoded polarization images as shown in $\mathbf{b}, \mathbf{c}, \mathbf{e}$, and $\mathbf{f}$, respectively.

\section{Methods}

Device fabrication. A GaN thin film with $1 \mu \mathrm{m}$ thickness is grown on a doubleside polished c-plane sapphire substrate using molecular beam epitaxy RIBER system. Conventional electron beam lithography processes are used for the etching of GaN nanopillars. The poly (methyl methacrylate) resist (495A4) with $\sim 180 \mathrm{~nm}$ is spin coated on the $\mathrm{GaN}$ and baked on a hot plate with temperature of $125^{\circ} \mathrm{C}$. It is exposed with designed patterns at $20 \mathrm{keV}$ (Raith ElphyPlus, Zeiss Supra 40) and developed in 3:1 IPA : MIBK solution. Oxygen plasma etching (reactive ion etching (RIE), Oxford System) is used to clean the residual resist that is not completely removed during development. The sample is deposited with a $50 \mathrm{~nm}$ thickness of Nickel using E-beam evaporation and is immersed into acetone solution for lift-off process to obtain the Nickel hard mask. By using RIE (Oxford System) with a plasma composed of $\mathrm{Cl}_{2} \mathrm{CH}_{4} \mathrm{Ar}$ gases, the pattern is transferred to the GaN. The residual nickel hard mask is removed by chemical etching with $1: 1$ $\mathrm{H}_{2} \mathrm{O}_{2}: \mathrm{H}_{2} \mathrm{SO}_{4}$ solution, revealing the GaN nanopillars. See more details of fabrication processes in Supplementary Fig. 1.

Conventional optical setup. The optical setup for characterizing the projected farfield is shown in Supplementary Fig. 2a. A laser beam propagates through a linear polarizer and a quarter waveplate (QWP). In order to avoid the birefringent effect of the sapphire substrate, we calibrate the polarization of the input light with a bare sapphire substrate to make sure the input light is LP in horizontal direction by controlling the rotation angle of the previous linear polarizer and QWP. After an achromatic lens with a focal length of $50 \mathrm{~mm}$, the laser beam is weakly focused on the metasurfaces. The first-order holographic image is projected onto a projector placed $10 \mathrm{~cm}$ away from the metasurface. A selected QWP with fast axis at the horizontal and a linear polarizer with axis of transmission at angle $\theta$ are used to analyze the images and measure the Stokes parameters.

Optical vectorial ptychography. Measurements were carried on a custom setup, based on an inverted microscope equipped with a motorized stage operating at 635 $\mathrm{nm}^{50,55}$. The measurement principle, which involves the recording of a series of far-field intensity patterns while scanning the specimen under a coherent illumination, is schematized in Supplementary Fig. 2b. All reported measurements in this work were obtained under the following specific parameters: the illumination probe was reduced to an effective diameter of $50 \mu \mathrm{m}$, by placing a $2 \mathrm{~mm}$ diameter iris diaphragm in the image plane of a $\times 40$ objective lens (ACHN-P, NA 0.65 , Olympus). The camera (Stingray F-145B, Allied Vision, $320 \times 240$ effective pixels of $25.8 \times 25.8 \mu^{2}$ after binning) was placed $190 \mathrm{~mm}$ after the diaphragm. Measurement scan was repeated for nine combinations of LP illumination $\left(0^{\circ}, 60^{\circ}\right.$, and $\left.120^{\circ}\right)$ and analysis $\left(0^{\circ}, 60^{\circ}\right.$, and $\left.120^{\circ}\right)$. Data processing was performed by means of a dedicated conjugate gradient algorithm ${ }^{56}$ running on a multi-graphics processor unit (DGX Station, NVIDIA), allowing the estimation of Jones matrices of the meta-holograms. The vectorial far field was obtained by modeling an LP illumination of the metasurface, followed by a propagation by fast Fourier transform of the vectorial exit field, as illustrated in Supplementary Fig. $2 c$. The spatial distribution of the propagation of the CV beams and two metasurface encryption are shown in Supplementary Movies 1-3.

\section{Data availability}

Ptychography raw data and other data that support the findings are available upon request to the authors.

\section{Code availability}

The code used for the vectorial meta-hologram design is available in the Supplementary Note 4 and Supplementary Data 1.

Received: 4 January 2021; Accepted: 20 May 2021;

Published online: 15 June 2021

\section{References}

1. $\mathrm{Yu}, \mathrm{N}$. et al. Light propagation with phase discontinuities: generalized laws of reflection and refraction. Science 334, 333-337 (2011).

2. Lalanne, P., Astilean, S., Chavel, P., Cambril, E. \& Launois, H. Blazed binary subwavelength gratings with efficiencies larger than those of conventional Échelette gratings. Opt. Lett. 23, 1081 (1998).

3. Genevet, P., Capasso, F., Aieta, F., Khorasaninejad, M. \& Devlin, R. Recent advances in planar optics: from plasmonic to dielectric metasurfaces. Optica 4 , 139 (2017).

4. Lalanne, P. \& Chavel, P. Metalenses at visible wavelengths: past, present, perspectives. Laser Photonics Rev. 11, 1600295 (2017).

5. Chen, H.-T., Taylor, A. J. \& Yu, N. A review of metasurfaces: physics and applications. Rep. Prog. Phys. 79, 076401 (2016).

6. Zheng, G. et al. Metasurface holograms reaching $80 \%$ efficiency. Nat. Nanotechnol. 10, 308-312 (2015).

7. Wang, S. et al. A broadband achromatic metalens in the visible. Nat. Nanotechnol. 13, 227-232 (2018).

8. Huang, L. et al. Three-dimensional optical holography using a plasmonic metasurface. Nat. Commun. 4, 2808 (2013).

9. Bomzon, Z., Biener, G., Kleiner, V. \& Hasman, E. Space-variant Pancharatnam-Berry phase optical elements with computer-generated subwavelength gratings. Opt. Lett. 27, 1141 (2002).

10. Yan, L. et al. $0.2 \lambda_{0}$ Thick adaptive retroreflector made of spin-locked metasurface. Adv. Mater. 30, 1802721 (2018).

11. Song, Q. H. et al. Split Archimedean spiral metasurface for controllable GHz asymmetric transmission. Appl. Phys. Lett. 114, 151105 (2019).

12. Lassaline, N. et al. Optical Fourier surfaces. Nature 582, 506-510 (2020). 
13. Deng, Z.-L. et al. Diatomic metasurface for vectorial holography. Nano Lett. 18, 2885-2892 (2018).

14. Deng, Z.-L. et al. Facile metagrating holograms with broadband and extreme angle tolerance. Light Sci. Appl. 7, 78 (2018).

15. Song, Q. et al. Ptychography retrieval of fully polarized holograms from geometric-phase metasurfaces. Nat. Commun. 11, 2651 (2020).

16. Arbabi, A., Horie, Y., Bagheri, M. \& Faraon, A. Dielectric metasurfaces for complete control of phase and polarization with subwavelength spatial resolution and high transmission. Nat. Nanotechnol. 10, 937-943 (2015).

17. Arbabi, E., Kamali, S. M., Arbabi, A. \& Faraon, A. Vectorial holograms with a dielectric metasurface: ultimate polarization pattern generation. ACS Photonics 6, 2712-2718 (2019).

18. Li, Y., Kreske, K. \& Rosen, J. Security and encryption optical systems based on a correlator with significant output images. Appl. Opt. 39, 5295-5301 (2000).

19. Han, J., Park, C.-S., Ryu, D.-H. \& Kim, E.-S. Optical image encryption based on XOR operations. Opt. Eng. 38, 47-54 (1999).

20. Tajahuerce, E., Matoba, O., Verrall, S. C. \& Javidi, B. Optoelectronic information encryption with phase-shifting interferometry. Appl. Opt. 39, 2313-2320 (2000).

21. Javidi, B. \& Nomura, T. Polarization encoding for optical security systems. Opt. Eng. 39, 2439-2443 (2000).

22. Unnikrishnan, G., Pohit, M. \& Singh, K. A polarization encoded optical encryption system using ferroelectric spatial light modulator. Opt. Commun. 185, 25-31 (2000).

23. Lippmann, G. Sur la théorie de la photographie des couleurs simples et composées par la méthode interférentielle. J. Phys. Theor. Appl. 3, 97-107 (1894).

24. Bryngdahl, O. \& Wyrowski, F. I Digital holography - computer-generated holograms. Prog. Opt. 28, 1-86 (1990).

25. Javidi, B. \& Nomura, T. Securing information by use of digital holography. Opt. Lett. 25, 28-30 (2000).

26. Huang, L., Zhang, S. \& Zentgraf, T. Metasurface holography: from fundamentals to applications. Nanophotonics 7, 1169-1190 (2018).

27. Genevet, P. \& Capasso, F. Holographic optical metasurfaces: a review of current progress. Rep. Prog. Phys. 78, 024401 (2015).

28. Deng, Z.-L. \& Li, G. Metasurface optical holography. Mater. Today Phys. 3 16-32 (2017).

29. Wan, W., Gao, J. \& Yang, X. Metasurface holograms for holographic imaging. Adv. Opt. Mater. 5, 1700541 (2017).

30. Deng, Z.-L., Zhang, S. \& Wang, G. P. A facile grating approach towards broadband, wide-angle and high-efficiency holographic metasurfaces. Nanoscale 8, 1588-1594 (2016).

31. Luo, X. et al. Integrated metasurfaces with microprints and helicity-multiplexed holograms for real-time optical encryption. Adv. Opt. Mater. 8, 1902020 (2020).

32. Zhao, R. et al. Nanoscale polarization manipulation and encryption based on dielectric metasurfaces. Adv. Opt. Mater. 6, 1800490 (2018).

33. Zhao, R. et al. Multichannel vectorial holographic display and encryption. Light Sci. Appl. 7, 95 (2018).

34. Deng, J. et al. Multiplexed anticounterfeiting meta-image displays with singlesized nanostructures. Nano Lett. 20, 1830-1838 (2020).

35. Khorasaninejad, M., Ambrosio, A., Kanhaiya, P. \& Capasso, F. Broadband and chiral binary dielectric meta-holograms. Sci. Adv. 2, e1501258 (2016).

36. Song, Q. et al. Bandwidth-unlimited polarization-mmaintaining metasurfaces. Sci. Adv. 7, eabe1112 (2021).

37. Chen, K. et al. Directional Janus metasurface. Adv. Mater. 32, 1906352 (2020).

38. Chen, Y., Yang, X. \& Gao, J. 3D Janus plasmonic helical nanoapertures for polarization-encrypted data storage. Light Sci. Appl. 8, 45 (2019).

39. Frese, D., Wei, Q., Wang, Y., Huang, L. \& Zentgraf, T. Nonreciprocal asymmetric polarization encryption by layered plasmonic metasurfaces. Nano Lett. 19, 3976-3980 (2019).

40. Shi, Y. et al. Chirality-assisted lateral momentum transfer for bidirectional enantioselective separation. Light Sci. Appl. 9, 62 (2020).

41. Yoon, G., Lee, D., Nam, K. T. \& Rho, J. "Crypto-display" in dual-mode metasurfaces by simultaneous control of phase and spectral responses. ACS Nano 12, 6421-6428 (2018).

42. Tang, Y. et al. Nonlinear vectorial metasurface for optical encryption. Phys. Rev. Appl. 12, 024028 (2019).

43. Deng, J. et al. Spatial frequency multiplexed meta-holography and metananoprinting. ACS Nano 13, 9237-9246 (2019).

44. Ren, H. et al. Metasurface orbital angular momentum holography. Nat. Commun. 10, 2986 (2019).

45. Fang, X., Ren, H. \& Gu, M. Orbital angular momentum holography for highsecurity encryption. Nat. Photonics 14, 102-108 (2020).

46. Ren, H. et al. Complex-amplitude metasurface-based orbital angular momentum holography in momentum space. Nat. Nanotechnol. 15, 948-955 (2020).

47. Li, J. et al. Addressable metasurfaces for dynamic holography and optical information encryption. Sci. Adv. 4, eaar6768 (2018).
48. Zhou, H. et al. Switchable active phase modulation and holography encryption based on hybrid metasurfaces. Nanophotonics 9, 905-912 (2020).

49. Kim, I. et al. Stimuli-responsive dynamic metaholographic displays with designer liquid crystal modulators. Adv. Mater. 32, 2004664 (2020).

50. Ferrand, P., Allain, M. \& Chamard, V. Ptychography in anisotropic media. Opt. Lett. 40, 5144 (2015).

51. Vyas, S., Kozawa, Y. \& Sato, S. Polarization singularities in superposition of vector beams. Opt. Express 21, 8972 (2013).

52. Zhan, Q. Cylindrical vector beams: from mathematical concepts to applications. Adv. Opt. Photonics 1, 1-57 (2009).

53. Schaefer, B., Collett, E., Smyth, R., Barrett, D. \& Fraher, B. Measuring the Stokes polarization. Parameters Am. J. Phys. 75, 163-168 (2007).

54. Rubin, N. A. et al. Matrix Fourier optics enables a compact full-Stokes polarization camera. Science 365, eaax1839 (2019).

55. Ferrand, P., Baroni, A., Allain, M. \& Chamard, V. Quantitative imaging of anisotropic material properties with vectorial ptychography. Opt. Lett. 43, 763 (2018).

56. Baroni, A., Allain, M., Li, P., Chamard, V. \& Ferrand, P. Joint estimation of object and probes in vectorial ptychography. Opt. Express 27, 8143 (2019).

\section{Acknowledgements}

We acknowledge funding from the European Research Council (ERC) under the European Union's Horizon 2020 research and innovation program (Grant agreement number 639109). P.F. acknowledges funding from the European Research Council (ERC) under the European Union's Horizon 2020 research and innovation program (Grant agreement number 724881). P.C.W. acknowledges the support from Ministry of Science and Technology, Taiwan (Grant numbers 108-2112-M-006-021-MY3 and 107-2923-M006-004-MY3), and the support from Ministry of Education (Yushan Young Scholar Program), Taiwan. P.C.W. also acknowledges the support in part by Higher Education Sprout Project, Ministry of Education to the Headquarters of University Advancement at National Cheng Kung University (NCKU).

\section{Author contributions}

Q.S. and P.G. conceived the idea and carried out the experiment. Q.S. performed the numerical simulation, calculation of meta-hologram, and optical characterization of meta-hologram. A.B. and P.F. performed the ptychography measurement, data processing, and analysis. Q.S., S.C., and V.B. contributed to the nano-fabrication. S.V., B.D., and P.M. performed the GaN MBE growth. Q.S., P.C.W., S.K., P.F., and P.G. wrote the manuscript. P.G. supervised and coordinated the project. All the authors contributed and approved the manuscript.

\section{Competing interests}

The authors declare no competing interests.

\section{Additional information}

Supplementary information The online version contains supplementary material available at https://doi.org/10.1038/s41467-021-23908-0.

Correspondence and requests for materials should be addressed to P.G.

Peer review information Nature Communications thanks Junsuk Rho and the other, anonymous, reviewer(s) for their contribution to the peer review of this work.

Reprints and permission information is available at http://www.nature.com/reprints

Publisher's note Springer Nature remains neutral with regard to jurisdictional claims in published maps and institutional affiliations.

\section{(1)}

Open Access This article is licensed under a Creative Commons Attribution 4.0 International License, which permits use, sharing, adaptation, distribution and reproduction in any medium or format, as long as you give appropriate credit to the original author(s) and the source, provide a link to the Creative Commons license, and indicate if changes were made. The images or other third party material in this article are included in the article's Creative Commons license, unless indicated otherwise in a credit line to the material. If material is not included in the article's Creative Commons license and your intended use is not permitted by statutory regulation or exceeds the permitted use, you will need to obtain permission directly from the copyright holder. To view a copy of this license, visit http://creativecommons.org/ licenses/by/4.0/.

(C) The Author(s) 2021 